\title{
V016 4FRONT - A PERSPECTIVE ON 4D SEISMIC METHODS
}

RICHARD C. UDEN and ADAM J.H. LITTLE

Scientific Software-Intercomp UK Ltd, Monarch House, Eversley Way, Egham Surrey TW2O $8 R Y$, UK

The underlying principle of $4 \mathrm{D}$ or time-lapse seismic is that production from a hydrocarbon reservoir causes changes to the reservoir fluid acoustic properties and fluid saturations as a function of time, which may be detected by repeat seismic surveys. In this paper we show the key elements of 4D seismic technology from the perspective of integration between static and dynamic reservoir models, drawing on experience gained in both full 4D projects and in pre-4D feasibility or scoping studies.

We begin by reviewing the framework for 4D seismic projects, and move onto the issues that need to be addressed when working on actual seismic data, how the elements of the scoping study are used to test the feasibility of a $4 \mathrm{D}$ project prior to acquisition of a repeat survey, and how the scoping study methods may subsequently be used in a comprehensive project. The part of the process that is currently of most interest concerns how the seismic measurement is used to update the reservoir engineering (i.e. simulation) model. We discuss the methods currently used and suggest improvements to the current methodology.

The principal benefit of 4D seismic to the reservoir management process is that it may provide an independent measurement of the relative position of gas and/or water flood fronts. The reservoir engineer uses the seismic measurement of fluid front location between the wells as an additional history matching parameter. Reservoir parameters controlling flow are modified to best match the seismic measurement, whilst honouring all of the other data traditionally used in history-matching. The enhancement thus gained in history-matching increases confidence in the reservoir model, therefore improving confidence in it's predictive capability.

The use of $4 \mathrm{D}$ seismic in history-matching of reservoir simulation models assumes that at least two compatible 3D surveys are available. It is frequently the case with either undeveloped or immature fields that a base 3D survey exists, and the option of subsequent surveys to be used as a reservoir management tool is under consideration. The main question here is "when will changes be sufficiently large to be detectable?". This question may be addressed by use of scoping studies run using reservoir simulation models.

The scoping study is based on forward modelling, and is used to test whether production-induced changes can be observed by a real seismic measurement given the constraints of the seismic system, mainly noise and bandwidth. Scenarios are designed to test the ability of the $4 \mathrm{D}$ method to detect, for example, oil bypassed by gas or water front advance.

In either history-matching or scoping study mode, reservoir simulation is used to compute the in-situ fluid property and saturation changes for a given scenario, which are then used to generate models of the seismic response, and thus the difference in seismic response. The strategy we follow is to use the seismic difference as the main measurement, and translate it to meaningful engineering information.

Maps of top reservoir amplitude as a function of time, for example, have previously been used to show seismic changes during production, but we stress the need to look at the whole reservoir and indeed areas 
outside of the reservoir. It pays not to have any preconceptions of what to expect from the seismic response during production.

A simulation model of an artificial reservoir is used to illustrate the methodology typically employed in modelling observed differences in $4 \mathrm{D}$ seismic response caused by production from the reservoir. The technology used is incorporated in a software package called 4FRONT, which has been, and is being, used on a number of projects. In general terms the package brings together reservoir engineering, petrophysics and geophysics. Geology is not forgotten but is normally included within petrophysics and geophysics.

The 4FRONT tools used to reconcile two seismic surveys are discussed. While it is desirable to use "designer" 3D surveys we advocate that the industry should make as much use as possible of existing 3D data. These have often been acquired before production started and therefore provide an excellent opportunity to be used as base surveys for $4 \mathrm{D}$ projects. The main processes used are survey rotation, wavelet matching and spatial registration and these are generally used in an iterative mode.

The principal goal for reconciliation is to minimise the differences between the two seismic surveys in a zone where no seismic changes are expected (subject to the preconception view stated above). This is typically sited above the reservoir in a gate large enough to obtain sufficient statistical data and small enough to minimise time variant effects.

The advantage of differencing this way is that the seismic amplitude variations due to lithology and porosity variations are assumed to cancel out in the differencing process leaving just the fluid change effects. Of course if the reservoir undergoes significant compaction due to pressure depletion during production then this complicates the picture.

When we come to making use of the seismic measurement to update the reservoir model we believe that the equivalence between depth and time surfaces is a critical factor. In order to know what reservoir layer you have information for you must know the time of the layer. Seismic inversion technology is a good way to generate this equivalence. It can be performed on either of the two surveys or perhaps the sum (and include the reservoir saturation data for the survey date) using a model derived from the reservoir data. It is important to note that the inversion works on layers not sampled data and therefore provides changes to the layer properties which is what is required by the reservoir model. In this way the depth surfaces in the reservoir model are updated to fit the seismic response. This is not peculiar to 4D seismic methods and can be done on regular 3D survey data.

The seismic difference can be interpreted for lateral extent and related to specific reservoir layers once the time to depth equivalence is made. The edges of the lateral extent of the difference can represent fluid front locations between the well control points. Approximate calibration of the seismic measurement of saturation may be made using production logs available from the wells.

Some aspects for the future include the use of $3 \mathrm{D}$ visualisation tools to help the integration of the reservoir model with the seismic difference volume and a direct seismic inversion of the difference volume. The seismic response to production is both in terms of amplitude changes at layer interfaces within the reservoir and as time delays below the reservoir which exhibit themselves as amplitude differences.

One of our main goals for the future is to use both the amplitude and time shift information in a seismic inversion for the reservoir interval to better define the vertical position of the observed changes, and to aid in quantifying the magnitude of the saturation change. 